\title{
Bayesian Juries and The Limits to Deterrence
}

\author{
Ezra Friedman and Abraham L. Wickelgren ${ }^{1}$
}

November 22, 2002

${ }^{1}$ Yale University and the Federal Trade Commission respectively. This work represents the views of the authors only and does not necessarily reflect the views of the Federal Trade Commission or any individual commissioner. We thank seminar participants at the 2002 North American Summer Meeting of the Econometric Society and at Yale University for comments. 


\begin{abstract}
We consider a model of crime with rational Bayesian Jurors. We find that if jurors are not perfectly informed, even when there is no limit to the size of the punishment that can be imposed, it is not possible to deter all crime. There is a finite lower bound on the crime rate which results from the difficulties in achieving a conviction with imperfect evidence and very low crime rates. Crime can not be reduced below this rate by increasing the penalty, but the lower bound can be decreased by improving the quality of evidence presented to jurors, or by increasing the threshold of evidence necessary for prosecution.

JEL Codes: K4,D8
\end{abstract}

Key Words: Bayesian Juries Deterrence Crime Punishment 


\section{Introduction}

Despite the recent decline in crime, a great many Americans (sometimes over 50 percent) view crime as the most important problem facing the United States (National Center for Policy Analysis 1999). Recent research has estimated that the social costs of crime are in excess of $\$ 1$ trillion (Anderson 1999), suggesting that such concern is quite reasonable. While, beginning with Gary Becker's (1968) seminal contribution, economic theory has made significant progress in understanding optimal crime control policies, it has yet to provide a satisfactory explanation for why society continues to be burdened by high crime rates and the tremendous costs they impose.

The most simple economic models of crime suggest that a rate of punishment can be set that would cheaply deter almost all inefficient crime. While acknowledging that detecting crime is costly, Becker argues that larger punishments can substitute for large probabilities of detection. Many previous papers have relaxed various assumptions of the Becker model in order to explain various features of crime and punishment in society. Much of this literature aims to explain why we do not see maximal punishments and very small detection rates (Polinsky and Shavell 1979; Posner 1985; Kaplow 1990; Malik 1990; Miceli 1990; Miceli 1991; Polinsky and Shavell 2000; Garoupa 2001), as Becker's theory suggests is optimal, and why fines do not play the large role in punishing criminals as Becker argues they should (Polinsky and Shavell 1984; Chu and Jiang 1993; Levitt 1997). See Garoupa (1997) and Polinsky and Shavell (2000) for excellent surveys of this literature.

While these papers have all added many valuable insights to our understanding of optimal crime control policies, as Reinganum and Wilde (1986) have pointed out, the implication of most of these models that the optimal crime rate should be very small is inconsistent with observed crime rates. In fact, most of the arguments as to why punishments should be strictly less than maximal depend on the existence of efficient crime in equilibrium (Reinganum and Wilde 1986). In his model of the optimal use of non-monetary sanctions, Shavell (1987) obtains a positive equilibrium crime rate (in his terminology, undesirable acts are committed) when sanctions are costly, some criminal activity is efficient, and courts cannot perfectly distinguish between efficient and inefficient crime. While this is undoubtedly explains why we do not completely deter some criminal acts, it is a less satisfactory explanation for serious violent crimes. For most serious crimes (e.g., rape) it is hard to believe that anything other than a vanishingly small percentage of it is efficient. In fact, Posner (1985, p.1195) argues that one can "derive the basic criminal prohibitions from the concept of efficiency;...what is forbidden is a class of inefficient acts." Furthermore, it implies a substantial 
portion of existing crime is efficient (otherwise, why not just sacrifice some efficient crime to deter inefficient crime and save on punishment costs). This is inconsistent with the tremendous social costs of crime discussed above.

Many authors also get positive equilibrium crime by assuming an exogenous upper bound on the magnitude of punishment. But, as Reinganum and Wilde (1986, p.3) point out, "the notion of an exogenous technological constraint on the extent of feasible sanctions seems inappropriate: we want to explain limited sanctions, not assume them. We do not inflict cruel and unusual punishment, but that is a statement about preferences, not technology." Other refinements have noted that, if there is some probability of legal error, infinite punishments (as long as they are not socially costless) that deter all crime will not be optimal since they would only be imposed on the innocent. While this argument does explain why crime exists in equilibrium, it also suggests that a large proportion of current convicts are innocent, something we are reluctant to believe. However this argument does capture the insight that as deterrence becomes more effective, a greater percentage of defendants are likely to be innocent.

The degree of social concern about crime suggests that few, if any, people believe that most crime is socially efficient, or that most convicts are innocent. Furthermore it suggests that society would support increased punishments if they were effective in deterring crime. This does not mean, however, that the economic model of crime is incapabable of explaining why we must face the large social costs associated with crime that we face today. We show that by introducing one more rational actor, juries, into the economic model of crime, we can explain why it is impossible to deter inefficient crime, even if all crime is both rational and inefficient. Furthermore, we obtain this result without the implication that a large fraction of current convicts must be innocent.

We show that if a jury convicts a defendant if and only if its posterior probability that the defendant is guilty exceeds some given level, then the accuracy of a jury's signal that a crime occurred provides a lower bound on the feasible crime rate. That is, so long as there is no signal that guarantees a crime occurred with probability one, it is impossible to eliminate crime, even if the punishments are infinitely costly to convicted defendants. Imagine an equilibrium with zero crime but imperfect signals of guilt. If the jury believes that it is never rational for anyone to commit a crime (because the expected punishment is so large), then without a perfect signal that a crime occurred it will never convict a defendant (the posterior probability of guilt is zero whenever the prior probability is zero unless the signal is perfect). But if no one is ever convicted, then crime is rational, undermining this equilibrium. Imperfect signals create a lower bound on the 
crime rate, even with infinite punishments and fully rational criminals.

One consequence of this relationship is that increasing the magnitude of punishment has a very small effect on the crime rate when the punishment is already quite large. While this effect is always positive when the threshold probability of guilt for conviction is independent of the punishment (so long as there is no atom at the top of the signal distribution), if this threshold is increasing in the magnitude of the punishment, as suggested by Andreoni (1991), then larger punishments can actually increase the crime rate.

An additional implication is that the minimum possible crime rate is determined by the accuracy of the most informative signal that the defendant is guilty. This suggests that improving the investigation of crime can reduce the crime rate more than increasing punishments. Specifically, reducing the likelihood that innocent defendants receive the most informative signal of guilt can be a most effective way of reducing the minimum possible crime rate. This suggests that credible prevention of prosecutorial misconduct is a particularly important element in the fight against crime, because the possibility of a coerced confession or planted physical evidence casts doubt on the most reliable signals of guilt.

All of these results rely on the assumption that there is at least some uncertainty in a trial about whether or not a crime has occurred, not just about who the guilty party is. While in some criminal cases the primary question is one of identity, in many cases the primary question is whether there was a crime. In drunk driving cases, for example, (or almost any driving related crime for that matter) the important uncertainty is typically over whether a crime was committed (e.g., was the driver drunk), rather than the identity of the driver. The same is typically true for fraud or other white collar crimes. Any claim of self-defense or insanity is an argument that no crime occurred. Rape defendants often make the argument no crime occurred because there was consent. A defendant in a theft cases can argue that the "stolen" item was a gift or belonged to the defendant for some other reason. Anytime arguments such as this can be made, and they cannot be proven wrong with probability one, the results in this paper apply: infinitely large punishments cannot eliminate crime (even when all potential criminals are rational), larger punishments reduce the probability of conviction and these punishments are imposed with positive probability.There are some other papers whose models do explain the existence of a substantial level of crime in equilibrium. Reinganum and Wilde's (1986) explanation is that the government lacks commitment power: punishment is costly and is only imposed after a crime has been committed, at which point it is too late to deter that crime. As a result, while it is optimal to threaten large punishments, it is 
not optimal to actually impose them ex post. Because criminals know this, threatening very large punishments will not deter all crime. Andreoni's (1991) paper also suggests a possible explanation for why the deterrent effect of punishment may be bounded. In his model, the larger the punishment the more confident the jury must be of the defendant's guillt for it to vote to convict. The main result is that a finite punishment may maximize deterrence. While both Andreoni's paper and our own focus on the role of jury decision-making, both the results and what drives them are quite different. Andreoni's paper shows that non-maximal punishments could be optimal because jury preferences may lead to a decreasing relationship between punishment magnitude and probability of conviction. In our paper, we find a limit to the effectiveness of deterrence, but what drives our results is simply Bayesian inference. We do not need to assume that juries are exogenously less likely to convict when punishment is increased. In fact, we simply assume that juries follow a simple fixed legal standard, vote to convict if and only if the probability of guilt is at least $\mathrm{P}$. Thus, we derive a positive equilibrium crime rate by only assuming that jurors are rational Bayesian updaters. It is also this paradigm that generates our results about the relative importance of criminal investigation and prosecutorial misconduct, results that are not present in models based on jury preferences or imperfect commitment.

Section two describes the model and contains the main results of the paper. Section three discusses possible extenstions and concludes.

\section{Model}

We asssume that there are a continuum of potential criminals. Each potential criminal receives a benefit $b$ (a random variable) from committing a crime. We assume $b \in B \subseteq \Re$ and let $h$ and $H$ be, respectively, the probability density function and cumulative distribution function for $b$. A trial occurs with a probability $q_{Y} \in(0,1)$ if a crime occurs, and with probability $q_{N} \in(0,1)$ if no crime has occured. In trials where a crime has actually occured, the likelihood that the prosecution has chosen the correct suspect is $m \in(0,1)$.

When a defendant is charged with a crime and brought to trial, the jury receives a signal of the defendant's guilt $t \in T \subseteq \Re$. This signal encompasses all the information revealed during the trial. The probability density function and cumulative distribution function for the signal are respectively given by $g_{G}$ and $G_{G}$ if the defendant is guilty and $g_{I}$ and $G_{I}$ if she is innocent. Without loss of

generality we assume $\frac{g_{G}(t)}{g_{I}(t)}$ is increasing in $t$, so a higher signal is a stronger signal of guilt. We 
also define a normalized signal $s \in S \subseteq[0,1]$ implicitly by the equation $\frac{g_{G}(t)}{g_{I}(t)}=\frac{s}{1-s}$. Thus, $s$ is the posterior probability of guilt if the prior is one-half. For most of the following analysis, we will work with the normalized signal.

We model jurors as rational Bayesian decision-makers. We abstract away from any issues of aggregation of information or heterogeneity in jurors and assume that jurors have identical information and preferences, and thus act monolithically. At the trial, they observe the signal $t$, and vote to convict if the defendant's posterior probability of guilt exceeds some threshold level, $P$. If the posterior probability of guilt is less than $P$ then the jury acquits the defendant. If the posterior probability is exactly $P$, then the jury may vote to convict or acquit. The threshold $P$ can either be thought of as determined by the jury's balancing of the costs of acquiting a guilty defendant and the costs of convicting an innocent one or as the level prescribed by law ( $P$ would then correspond to "reasonable doubt").

Because jurors are rational Bayesians, we will require the prior probability of guilt to be consistent with the crime rate. The prior probability that an individual on trial is guilty will be referred to as $\mu$, and it is a function of the crime rate and the variables $q_{Y}, q_{N}$, and $m$. If the crime rate is $C$, so the likelihood there has been no crime is $1-C$, the prior probability of guilt is as follows.

$$
\mu=\frac{m q_{Y} C}{q_{Y} C+q_{N}(1-C)}
$$

Differentiating $\mu$ with respect to $C$, we obtain $\frac{d \mu}{d C}=\frac{m q_{Y} q_{N}}{\left(q_{Y} C+q_{N}(1-C)\right)^{2}}$. Note that as long as $q_{Y}>0$ and $q_{N}>0$, the prior $\mu$ is increasing in the crime rate and is zero when the crime rate is zero.

The probability density functions and cumulative distribution functions for the normalized signal are given by $f_{G}$ and $F_{G}$ if the defendant is guilty and $f_{I}$ and $F_{I}$ if she is innocent. Using the normalized signal, the posterior probability, $\rho$, that a defendant with a signal $s$ is guilty is given by:

$$
\rho=\frac{\mu s}{\mu s+(1-\mu)(1-s)}
$$

Given the prior probability $\mu$, a jury will convict a defendant if and only if the defendant's signal of guilt is large enough. This cutoff value for conviction is given by setting the right hand 
side of (2) equal to $P$ and solving for $s$. We call this cutoff value $\widehat{s}$.

$$
\widehat{s}(\mu)=\frac{P(1-\mu)}{P(1-\mu)+\mu(1-P)}
$$

Clearly, $\widehat{s}$ is decreasing in $\mu$, meaning that with a higher prior of guilt, the jury requires a weaker signal to convict. Since $\mu$ is increasing in the crime rate, a lower crime rate implies that the jury requires a stronger signal to convict. As the crime rate and the prior probability go to zero, the signal required for conviction goes to one. Now, let $s_{M}=S u p\{s \in S\}$. From (3), it is easy to see that the lowest possible prior probability that is consistent with a jury sometimes convicting a defendant is when the jury convicts only if it receives the strongest signal of guilt, $s_{M}$. The lower bound on the prior probability is then given by solving (3) for $\mu$ with $\widehat{s}=s_{M}$.

$$
\mu_{M}=\frac{P\left(1-s_{M}\right)}{P\left(1-s_{M}\right)+s_{M}(1-P)}
$$

This minimum prior corresponds to a lower bound on the crime rate, below which it is impossible for a rational jury to achieve reasonable doubt. We can compute this by inverting (1) to obtain crime rate as a function of the prior, and setting the prior equal to the right hand side of (4) for an arbitary $s$.

$$
\hat{C}(s)=\frac{q_{N}}{m q_{Y} \frac{s}{1-s} \frac{1-P}{P}+(m-1) q_{Y}+q_{N}}
$$

This is decreasing in $s$, so the minimum crime rate consistent with a conviction by a rational jury is found by setting $s=s_{M}$.

$$
C_{M}=\frac{q_{N}}{m q_{Y} \frac{s_{M}}{1-s_{M}} \frac{1-P}{P}+(m-1) q_{Y}+q_{N}}
$$

Now consider an individual's incentive to commit a crime. A person commits a crime if the benefit, $b$, exceeds the expected cost from being convicted and punished by an amount $z$. Notice that in our model, the possibility of wrongful conviction has no direct impact on an individual's decision to commit a crime. We believe this is realistic for three reasons. First, even if deciding not to commit a given crime increases the probability that this person will be convicted of a crime for which she is innocent, this probability is, in most cases, vanishingly small. Even if innocent people get convicted quite often, she is just one of a very large number of innocent people who could be wronfully convicted. Second, and more importantly, as was pointed out by Schrag and Scotchmer 
(1994), crime opportunities can be either exclusive or non-exclusive. If a crime opportunity (or the benefit from that crime) is exclusive, then if one does not commit this crime, no one will. Not committing the crime does not increase one's probability of being wrongfully convicted of a crime. Thus, in the real world this assumption is approximately correct for non-exclusive crimes and exactly correct for exclusive crimes. Third, assuming away any direct effect on the crime rate from the possibility of wrongful convictions highlights the result in Proposition 2 that reducing the risk of wrongful convictions will, in fact, indirectly reduce the crime rate.

According to our model, the probability of being convicted of a crime is given by $m q_{Y}\left(1-F_{G}(\widehat{s})\right)$. Our assumption is that an individual commits a crime if and only if $b>z m q_{Y}\left(1-F_{G}(\widehat{s})\right)$. Given this decision rule, the probability that an individual's benefit from crime is large enough to induce her to commit a crime is $1-H\left(z m q_{Y}\left(1-F_{G}(\widehat{s})\right)\right)$. If juries have rational expectations, this crime rate must be consistent with the prior probability of guilt that is consistent with the cutoff value of the signal necessary for conviction, $\widehat{s}$. That is:

$$
1-H\left(z m q_{Y}\left(1-F_{G}(\widehat{s})\right)\right)=C(\widehat{s})=\frac{q_{N}}{m q_{Y} \frac{\hat{s}}{1-\hat{s}} \frac{1-P}{P}+(m-1) q_{Y}+q_{N}}
$$

These last two equalities are the basis for the following proposition.

Proposition 1. (a) The greater the punishment for crime, the lower is the probability of conviction. (b) If $f_{G}$ contains an atom at $s_{M}$ then there exists a $Z<\infty$, such that for all $z>Z$, increasing the punishment, $z$, has no effect on the crime rate $C$. (c) If $s_{M}<1$ (there is no signal that a guilty person will receive with positive probability that an innocent person will never receive), then the minimum possible crime rate is strictly positive no matter how large the punishment.

Proof: (a) Totally differentiate (7) with respect to $\mathrm{z}$ and solve for $d \widehat{s} / d z$ :

$$
d \widehat{s} / d z=\frac{m q_{Y} q_{N}}{\left(q_{Y} R+q_{N}(1-R)\right)^{2}} \frac{\left(1-F_{G}(\widehat{s})\right) h\left(z\left(1-F_{G}(\widehat{s})\right)\right.}{\{P(1-P) /[P(1-\widehat{s})+\widehat{s}(1-P)]\}+z f_{G}(\widehat{s}) h\left(z\left(1-F_{G}(\widehat{s})\right)\right.} \geq 0
$$

Since the probability of conviction is $1-F_{G}(\widehat{s})$, the result follows.

(b) If $f_{G}$ has an atom at $s_{M}$, then $1-F_{G}\left(s_{M}\right)=f_{G}\left(s_{M}\right)>0$. Hence, there is a finite $Z$ such that:

$$
1-H\left(Z m q_{Y} f_{G}\left(s_{M}\right)\right)=\frac{q_{N}}{m q_{Y} \frac{s_{M}}{1-s_{M}} \frac{1-P}{P}+(m-1) q_{Y}+q_{N}}
$$

The right hand side of (8) is the minimum crime rate consistent with any convictions. Hence, 
increasing $z$ above $Z$ cannot decrease the right hand side further. By this equality, then, the crime rate cannot fall below this level. The equality can only be maintained by decreasing the probability of conviction for $z>Z$. This can occur for a fixed $f_{G}$ if jurors play a mixed conviction strategy when observing $s_{M}$. That is, the larger is $z>Z$, the lower is the probability of conviction when jurors observe $s_{M}$. Thus, for $z>Z$, the argument in the $H$ function on the left hand side of $(7)$ becomes $z q_{Y} \hat{f}_{G}\left(s_{M}, z\right)$, where $\widehat{f}_{G}(s, z)=f_{G}(s)$ for $s<s_{M}$ and $\widehat{f}_{G}\left(s_{M}, z\right)$ satisfies $\left(z m q_{Y} \widehat{f}_{G}\left(s_{M}, z\right)\right)=\frac{P\left(1-s_{M}\right)}{P\left(1-s_{M}\right)+s_{M}(1-P)}$.

(c) The right hand of (6) is strictly positive for any $s_{M}<1$ as long as $q_{N}>0$. Since this is the minimum of the right hand side of ( 7$)$, it is also the minimum of the left hand side. Thus, there is a strictly positive probability that an individual will find it optimal to commit a crime, making the minimum possible crime rate positive. Q.E.D.

Part (a) of the Proposition says that increases in punishment will have a smaller effect on the crime rate than in the standard model without juries. In the standard model, an $x$ percent increase in the punishment magnitude increased the required benefit from crime by the marginal criminal by $x$ percent. In our model, the required marginal benefit increases by less than $x$ percent because the likelihood of conviction falls when the punishment increases. Since the required benefit increases less, the deterrent effect from increased punishment is smaller than when juries are ignored. This result is similar to that of Andreoini (1991), but, unlike Andreoni, we get this result while holding the conviction standard, $P$, constant.

As part (b) indicates, when $P$ is held constant, we do not get Andreoini's result that larger punishments can increase the crime rate. If there is an atom at the top of the signal distribution, however, it will be the case that there is a finite punishment that yields the minimum crime rate. Punishment levels above that decrease the conviction rate so that the expected punishment is held constant. If there is no atom at the top of the signal distribution, then an infinite punishment is necessary to push the crime rate to its minimum level. Any finite punishment will lead to a signal cutoff for conviction that is strictly less than the maximum signal, $\widehat{s}<s_{M}$, so that (7) is satisfied.

Part (c) explains why the policy of setting punishments so large that all crime is deterred (and so the cost of punishment never becomes an issue) is not feasible. There can never be an equilibrium with no crime because then the jury's prior precludes conviction (unless it can receive a perfect signal of guilt). In a rational model of crime, if crime is never rational and juries know this then, absent a perfect signal, the evidence can never be strong enough to warrant conviction. Since some 
crime is inevitable in equilibrium, the cost of punishment matters. So, if this cost is increasing in the magnitude, finite punishment is optimal. Note that we do not preclude a finite upper bound on $b$, so our results do not depend the on the presence of criminals whom it is impossible to deter from crime.

As the following proposition demonstrates, this model also shows that protections against wrongful conviction play an important role in deterring crime.

Proposition 2. Say the original normalized signal required for conviction is $\widehat{s}$ corresponding to the original signal $\widehat{t}$, i.e., $\frac{g_{G}(\widehat{t})}{g_{I}(\hat{t})}=\frac{\widehat{s}}{1-\widehat{s}}$. Reducing the likelihood that the jury receives a signal $t^{\prime}$ for all $t^{\prime}$ in a neighborhood of $\widehat{t}$ when the defendant is innocent will reduce the crime rate.

Proof: The crime rate is determined by $1-H\left(z q_{Y}\left(1-G_{G}(\widehat{t})\right)\right)$, the probability that a person finds it optimal to commit a crime given the punishment, $z$, and the probability of conviction, $1-G_{G}(\hat{t})$. Reducing the probability that an innocent person receives a signal $t^{\prime}$ will reduce the crime rate if and only if it leads to a reduction in $\widehat{t}$. Of course $\widehat{t}$ is determined by the analogue of equation (7):

$$
1-H\left(z m q_{Y}\left(1-G_{G}(\hat{t})\right)\right)=\frac{q_{N}}{m q_{Y} \frac{g_{G}(\hat{t})}{g_{I}(\hat{t})} \frac{1-P}{P}+(m-1) q_{Y}+q_{N}}
$$

We use the parameter $\lambda$ to capture the accuracy of the signal. In particular, we assume that an increase in $\lambda$ corresponds to a reduction in $g_{I}(\widehat{t})$, that is $\frac{\partial g_{I}(\widehat{t}, \lambda)}{\partial \lambda}<0$. The right side of (9) is clearly increasing in $g_{I}(\widehat{t})$, furthermore the left side is increasing in $\widehat{t}$ and the right side is decreasing in $\widehat{t}$ (by our MLRP assumption for $G$ ). Therefore, reducing $g_{I}(\widehat{t})$ causes the left side to be greater than the right. To restore equality we must reduce $\hat{t}$. This decreases the crime rate, represented by the left side of the equation. Q.E.D.

While many authors have made the point that reducing the probability of wrongful convictions can increase deterrence, this was under the implicit assumption that crime opportunities are nonexclusive. Thus, the probability of wrongful convictions represent a cost to not committing a crime. Proposition 2 shows that even without this effect reducing the probability of wronful convictions reduces the crime rate because it increases the probability that a jury will convict a guilty defendant. This result is somewhat related to the result in Schrag and Scotchmer (1994) that when police are prejudiced against habitual criminals (in our context, they are more likely to receive high signals), juries will require higher signals to convict, inducing them to commit more crimes.

Proposition 2 shows that increasing the quality of evidence presented at trial will reduce the 
crime rate. In addition to increasing the quality of evidence, however, it may also be possible for the prosecution to improve the process by which it chooses when to have a trial and which defendant to try. The next proposition shows that any unambiguous improvement in this process will also decrease the crime rate.

Proposition 3. Increasing $q_{Y}$, the likelihood of a trial when there is a crime, decreasing $q_{N}$, the likelihood of a trial if there is no crime or increasing $m$, the likelihood of trying the correct defendant, decreases the crime rate.

Proof: Differentiating (7) we see that the right side is increasing in $q_{N}$, decreasing in $q_{Y}$ and decreasing in $m$. The left side is decreasing in $m$ and $q_{Y}$ and does not depend directly on $q_{N}$. Increasing $q_{N}$ increases only the right side of the equation, implying that $\widehat{s}$ must increase in order for the equation to be satisfied, which requires an increase in the crime rate on the left side of the equation. Increasing $q_{Y}$ or $m$ decreases both sides of the equation. Let $\hat{s}^{\prime}$ be the new value at which the equation is satisfied. Notice that the right hand side is decreasing in $\hat{s}$ and the left hand side is increasing in $\widehat{s}$. Say $\widehat{s}$ is enough greater than $\widehat{s}$ that the crime rate increases. This means the left hand side must increase. But, since $\widehat{s}$ increases and $q_{Y}$ or $m$ increases, the right hand side must decrease. This contradiction implies that (7) must be satisfied at a lower crime rate than before. Q.E.D.

The results in proposition 3 imply that an improvement in the process by which the prosecution chooses when to prosecute, or an improvement in the decision of which individual to prosecute, will decrease the crime rate. Unlike the decrease in crime from increasing punishments, the effects of changing these parameters do not rapidly reach an asymptote. This may suggest that once punishments are sufficiently severe, resources devoted to improving the prosecutorial process would have a greater impact on crime than resourse devoted to increasing the severity of the punishment.

According to Proposition 3, decreasing $q_{N}$ decreases crime, while decreasing $q_{Y}$ increases crime. In practice it might be very difficult to prosecute less innocent defendants without prosecuting less guilty defendants as well, that is to say a decrease in $q_{N}$ may always entail some decrease in $q_{Y}$. Therefore, it is worthwhile to examine the trade off inherent in more or less agressive prosecution. We consider how much of a decrease in $q_{N}$ would be enough to make a decrease in $q_{Y}$ worthwhile. The following proposition provides our results regarding this trade off.

Proposition 4. Consider two pairs of parameters $\left\{q_{N}, q_{Y}\right\}$ and $\left\{q_{N}^{\prime}, q_{Y}^{\prime}\right\}$ If $s_{M}<1$ and $\frac{q_{N}^{\prime}}{q_{Y}^{\prime}}<\frac{q_{N}}{q_{Y}}$ then there is some $\widehat{z}$ such that if $z>\widehat{z}$ crime is lower with $\left\{q_{N}^{\prime}, q_{Y}^{\prime}\right\}$ than with $\left\{q_{N}, q_{Y}\right\}$ 
Proof: Because both sides of (7) must be positive (the equilibrium crime rate cannot be zero), $\lim _{z \rightarrow \infty} F_{G}(\widehat{s}(z))=1$. Likewise $\lim _{z \rightarrow \infty} F_{G}\left(\widehat{s}^{\prime}(z)\right)=1$. This implies $\lim _{z \rightarrow \infty} F_{G}(\widehat{s}(z))=$ $\lim _{z \rightarrow \infty} F_{G}\left(\widehat{s}^{\prime}(z)\right)=F_{G}\left(s_{M}\right)$.

By (7), this implies that

$$
\lim _{z \rightarrow \infty} C(\widehat{s}(z))=\frac{q_{N}}{m q_{Y} \frac{s_{M}}{1-s_{M}} \frac{1-P}{P}+(m-1) q_{Y}+q_{N}}=\frac{\frac{q_{N}}{q_{Y}}}{m \frac{s_{M}}{1-s_{M}} \frac{1-P}{P}+(m-1)+\frac{q_{N}}{q_{Y}}}
$$

and

$$
\lim _{z \rightarrow \infty} C\left(\widehat{s}^{\prime}(z)\right)=\frac{q_{N}^{\prime}}{m q_{Y}^{\prime} \frac{s_{M}}{1-s_{M}} \frac{1-P}{P}+(m-1) q_{Y}^{\prime}+q_{N}^{\prime}}=\frac{\frac{q_{N}^{\prime}}{q_{Y}^{\prime}}}{m \frac{s_{M}}{1-s_{M}} \frac{1-P}{P}+(m-1)+\frac{q_{N}^{\prime}}{q_{Y}^{\prime}}}
$$

Since $m \frac{s_{M}}{1-s_{M}} \frac{1-P}{P}+(m-1)>0$ (otherwise the crime rate is greater than one), this implies $\left.\lim _{z \rightarrow \infty} C(\widehat{s} z)\right)>\lim _{z \rightarrow \infty} C\left(\widehat{s}^{\prime}(z)\right)$,so with great enough punishment any decrease in $\frac{q_{N}}{q_{Y}}$ will decrease the crime rate. Q.E.D.

The results of proposition 4 show that if it is possible to sufficiently increase the punishment, crime can be minimized by minimizing $\frac{q_{N}}{q_{Y}}$. If we believe that reducing the agressiveness in prosecution would result in proportionately less prosecutions when there has actually not been a crime, this would imply that juries will be more likely to convict. In situations where it is difficult to obtain convictions because crime is near the lower bound, the increases in the likelihood of conviction given trial will outweight the reduction in the likelihood of a trial, and thus less agressive prosecution would actually reduce the crime rate. In essence, this proposition suggests that rather than aiming for more prosecutions, an effective anti-crime strategy would focus prosecutions on suspects who are most likely to be guilty, and push for severe punishments for those suspects.

\section{Conclusion and Extensions}

One simplification present in our model is that we assume jurors do not receive an independent signal of the likelihood that a crime actually occured. However, if juries did receive such a signal, as long as this signal is never perfect, the results of our model will still hold. In the new model, the posterior belief of the jury would be a function of the signal of the defendant's guilt as well as the signal of whether there had actually been a crime. The maximum probability that the jury would place on the defendant's guilt would occur when the jury receives the strongest possible signal that a crime occurred and the strongest signal of the defendant's guilt. This posterior probability would 
still be an increasing function of the crime rate. Since this probability would go to zero as the crime rate went to zero, this would imply that, as before, there is a minimum crime rate that is consistent with conviction. All the other results would be qualitatively similar. Another possible extension to our model would be to formally model the prosecution and the grand jury process. For example, we could assume that the prosecution received an independent signal of the suspects guilt, and could choose to prosecute only when their independent signal exceeded some cut-off level. Raising this cut-off level would have the effect of decreasing both $q_{N}$ and $q_{Y}$, but decreasing $q_{N}$ proportionately more. One could argue about whether or not the prosecution could credibly raise this cut-off level, since the prosecution usually has an incentive to prosecute and convict as many people as possible. However, even if the prosecution could credibly commit to a cut-off level, as long as there is an upper bound on the strength of the prosecution signal, our qualitative results would be unchanged. Even if it was believed that the prosecution only prosecuted suspect after receiving the strongest signal of guilt, as long signal could not be perfect, the jury's prior would still go to zero as the crime rate went to zero and there would be a positive lower bound on crime. In short we expect there will be a lower bound on the crime rate in any model where there is no perfect signal that a crime actually occurred.

It would be relatively easy to combine our model with Andreoni's (1991), where a jury requires a higher standard of proof when a punishment is more severe. Under our assumption that the quality of information presented to the jury is bounded, we would find that there was a punishment level associated with a minimum crime rate. Any increase in punishment above that level would require a standard of reasonable doubt that could not be reached or surpassed unless the crime rate increased, suggesting that crime rate plotted against punsihment would have a U-shaped graph. In this case, allowing for Bayesian juries would actually suggest that the crime rate increased with punishment more slowly than in the orignal Andreoni paper. The Bayesian jury will recognize that as they raise their reasonable doubt threshold, the crime rate will increase, and thus they will increase their priors on the defendants guilt, partially mitigating the increase in crime that Andreoni predicts.

Our model has some clear empirical predictions, which may be worthwhile to test. Specifically, our model suggests that increases in punishment severity are likely to result in fewer convictions, but are likely to have only a small effect on the actual crime rate. These predictions are in line with Andreoni's (1995) re-examination of the data from Ehrlich's 1973 study. Our model also predicts that the likelihood of conviction conditional on trial should be positively related to the crime rate, 
but conditional on the crime rate, the likelihood of conviction should be negatively related to the number of trials. Testing this relationship could be the subject of a future empirical study. However any cross-sectional or time series estimation of this relationship would have to account for differences in propensity to commit crime as well as differences in standards of reasonable doubt.

We have shown that when juries are rational Bayesians, increasing the punishment for crimes will have diminishing effects unless the jury can be perfectly sure that a crime occurred. In fact, the crime rate asymptotically approaches a strictly positive lower bound as the punishment approaches infinity. Our ability to deter crime is not limited so much by our inability or unwillingness to impose severe penalties, but rather by limitations on our ability to prove the guilt of any defendant. This suggests that rather than increasing punishment, improving the investigation of crime may be a more effective way to lower the crime rate. Furthermore, the results of this paper suggest that agressive prosecution can be couterproductive and that prosecutorial misconduct can be particularly harmful. The possibility of prosecutorial misconduct injects reasonable doubt into the beliefs of juries, and undermines the possibility of conviction with low crime rates. In short, accounting for rational Bayesian jurors shows that extreme punishments, even if they are feasible and socially acceptable, may be unable to solve our problems with crime. 


\section{References}

\section{References}

[1] Anderson, David A. "The Aggregate Burden of Crime" Journal of Law and Economics 42 (October 1999): 611-641

[2] Andreoni, James. "Reasonable Doubt and the Optimal Magnitude of Fines: Should the Penalty Fit the Crime" Rand Journal of Economics 22 (Autumn 1991): 385-95

[3] _-__.. "Criminal Deterrence in the Reduced Form: A New Perspective on Ehrlich's Study" Economic Inquiry 33 (July 1995): 476-83

[4] Becker, Gary S. "Crime and Punishment: an Economic Approach" Journal of Political Economy (April 1968): 169-217

[5] Chu, C.Y.Cyrus and Jiang, Neville. "Are Fines more Efficient than Imprisonment?" Journal of Public Economics 51 (July 1993): 391-413

[6] Ehrlich, Isaac. "Participation in Illegitimate Activities: A Theoretical and Empirical Investigation" Journal of Political Economy 81 (June 1973) 521-65

[7] Garoupa, Nuno. "The Theory of Optimal Law Enforcement" Journal of Economic Surveys 11(September 1997): 267-95

[8] Kaplow, Louis. "A Note on the Optimal Use of Nonmonetary Sanctions" Journal of Public Economics 42 (July 1990): 245-247

[9] Malik, Arun S. "Avoidance, Screening and Optimum Enforcement" Rand Journal of Economics 21 (Autumn 1990): 341-353

[10] Micelli, Thomas J. "Optimal Prosecution of Defendants Whose Guilt is Uncertain" Journal of Law, Economics and Organization 6 (Spring 1990): 189-201

[11] _-_-_. "Optimal Criminal Procedure: Fairness and Deterrence" International Review of Law and Economics 11 (May 1991): 3-10

[12] National Center for Policy Analysis. "Crime and Punishment in America: 1998" NCPA Policy Report No. 229. October 1999. 
[13] Polinsky, A. Mitchell and Shavell, Steven. "The Optimal Trade-Off between the Probability and Magnitude of Fines" American Economic Review 69 (December 1979): 880-891

[14] _____. "The Optimal Use of Fines and Imprisonment" Journal of Public Economics, 24 (June 1984): 89-99

[15] ____.. "The Economic Theory of Public Enforcement of Law" 38 Journal of Economic Literature 38 (March 2000): 45-76

[16] Posner, Richard A. "An Economic Theory of the Criminal Law" Columbia Law Review 85 (October 1985): 1193-1231

[17] Reinganum, Jennifer and Wilde, Louis. 1986 "Credibility and Law Enforcement" mimeo

[18] Schrag, Joel and Schotchmer, Suzanne. "Crime and Prejudice: The Use of Charcter Evidence in Criminal Trials" Journal of Law, Economics and Organization 10 (October 1994): 319-342

[19] Shavell, Steven. "The Optimal Use of Nonmonetary Sanctions as a Deterrent" American Economic Review 77 (September 1987): 584-592 